\title{
Gait cycle prediction model based on gait kinematic using machine learning technique for assistive rehabilitation device
}

\author{
Che Ani Adi Izhar, Z. Hussain, M. I. F. Maruzuki, Mohd Suhaimi Sulaiman, A. A. Abd. Rahim \\ Faculty of Electrical Engineering, Universiti Teknologi MARA, Malaysia
}

\begin{tabular}{l}
\hline \hline Article Info \\
\hline Article history: \\
Received Oct 31, 2020 \\
Revised May 5, 2021 \\
Accepted May 20, 2021 \\
\hline
\end{tabular}

\section{Keywords:}

Assistive rehabilitation

Devices

Gait cycle prediction

Machine learning

\begin{abstract}
The gait cycle prediction model is critical for controlling assistive rehabilitation equipment like orthosis. The human gait model has recently used statistical models, but the dynamic properties of human physiology limit the current approach. Current human gait cycle prediction models need detailed kinematic and kinetic data of the human body as input parameters, and measuring them requires special instruments, making them difficult to use in real-world applications. In our study, three separate machine learning algorithms were used to create a human gait model: Gaussian process regression, support vector machine, and decision tree. The algorithm used to create the model's input parameters are height, weight, hip and knee angle, and ground reaction force (GRF). For better gait cycle model prediction, the models produced were enhanced by incorporating different sliding window data. The best gait period prediction model was DT with sliding window data $(\mathrm{t}-3)$, which had a root mean square error of 3.3018 and the R-squared (R-Value) of 0.97. The projection model focused on hip and knee angle and GRF was a feasible solution to controlling assistive rehabilitation devices during the gait cycle.
\end{abstract}

This is an open access article under the $\underline{C C B Y-S A}$ license.

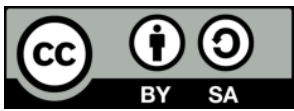

\section{Corresponding Author:}

Adi Izhar bin Che Ani

Faculty of Electrical Engineering

Universiti Teknologi MARA Cawangan Pulau Pinang

13500, Permatang Pauh, Pulau Pinang, Malaysia

Email: adiizhar@uitm.edu.my

\section{INTRODUCTION}

Human gait rehabilitation is the process of regaining patient mobility from paralyzed muscles caused by neurological disorders such as stroke and SCI [1]. The technique in human gait rehabilitation includes orthosis [2], [3], and functional electrical stimulation (FES) [4], [5] as the assistive device to help the rehabilitation process. Gait analysis is the biomedical study of the lower limbs during locomotion [6]. Medical practitioners often face the daunting prospects of storing and analyzing vast volumes of data typically obtained during several gait cycles. For example, Chen et al. [7] highlighted the difficulties faced by doctors in assessing quantitative analysis of gait anomalies, thus developing a gait acquisition and analysis method for osteoarthritis prediction model using RGB-D camera. In gait rehabilitation, researchers such as Galli et al. [8] and Vallery et al. [9] have used the data collected during gait rehabilitation for the purpose of exoskeleton control designs to suit patient needs during rehabilitation. The data collection of gait study used technique such as optical system, electromyography, goniometric system, and imaging [10]-[13]. Quantification of walking parameters by the gait cycle is important for a deeper understanding of human locomotion and for the regulation of assistive devices. A gait can be divided into stance and swing phases, separated by initial contact and foot-off events as 
shown in Figure 1. The stance and swing phases are divided into $60 \%$ and $40 \%$ of the gait cycle. Gait patterns can be recognized from kinetic and kinematic parameters during gait progression.

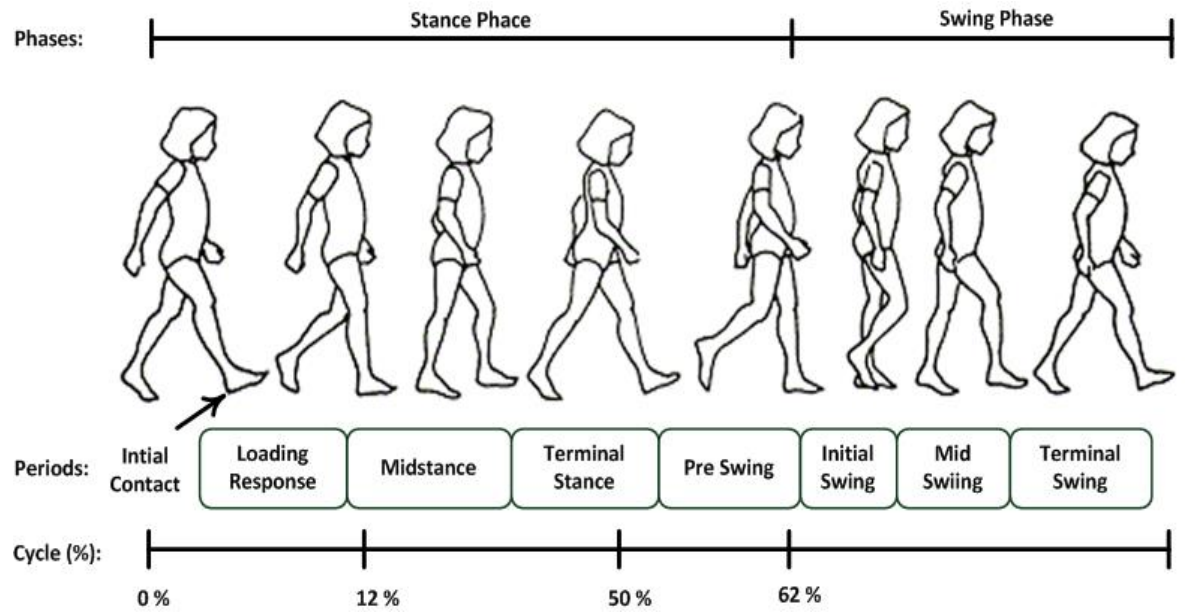

Figure 1. Gait cycle phases [13]

Research conducted by Farah et al. [14] determined gait phase detection can be implemented by using machine learning techniques. The parameters used in the study were knee angle, thigh angular velocity, and thigh center of gravity acceleration for all planes of gait progression. The study was done in the laboratory setting, with more than $97 \%$ accuracy for the identification of gait events. However, the used of all three sagittal, coronal, and transverse planes of gait progression for detection of gait cycle may lead to difficulties in usage of the detection model. On the other hand, despite using the above-mentioned parameters, Mahdavian et al. [15], developed the model for lower limb motion by using machine learning techniques from ground reaction forces (GRF) parameter. The model developed successfully predicted the user's gait steps with more than $80 \%$ accuracy, which was then incorporated with the hip exoskeleton control scheme. However, the detected GRFs value at all planes of gait regression may not identically represent the gait cycle itself. Susanto et al. [16], investigated the use of artificial neural network (ANN) for a prediction of the assistive torque required for lower limb exoskeleton from previous human walking gait cycles and the center of pressure $(\mathrm{CoP})$. The study successfully controlled the lower limb exoskeleton from the prediction of ANN on the different walking speed situations. It is suggested that more input variables should be given to ANN so that better prediction models can be developed. Another research by Young et al. [17] and Kilmartin et al. [18] used dynamic Bayesian networks (DBN) to determine the current state and make a prediction of what the next state will be, which takes into account previous data and states. Subject-independent algorithms that include generalized gait and transition prediction classification have also shown promise for both able-bodied subjects and users of prostheses. In the laboratory setting, these algorithms were highly accurate, with greater than $95 \%$ accuracy for the detection of gait events. Building on the success of previous work stated above, artificial intelligence algorithms have been put in place to leverage the temporal nature of gait data and to optimize the classification or prediction of gait events. These algorithms require large sensory arrays, i.e., multiple mechanical and neuromuscular sensors for classification and predictions. From these scenarios it seems that the prediction of gait cycle during gait progression is vital and better control schemes can be developed for rehabilitation assistive devices such as orthosis and exoskeleton. The goal of this research work is to predict the gait cycle using the selected features that includes knee angle, hip angle and GRF at sagittal plane using machine learning algorithms. In this work, Gaussian process regression (GPR), support vector machine (SVM) and decision trees (DT) were used and compared to predict gait cycle.

\section{RESEARCH METHOD}

The studies conducted in this research included developing the gait cycle prediction model using three different machine learning algorithms that were GPR, SVM and DT. In order to develop the model, the study work was begun with data preparation for the training, parameter selection and sliding window, constructing the gait cycle prediction model and model performance evaluation via root means square error (RMSE) and RSquared (R-Value) values. Figure 2 shows the flowchart of work carried out in this research. 


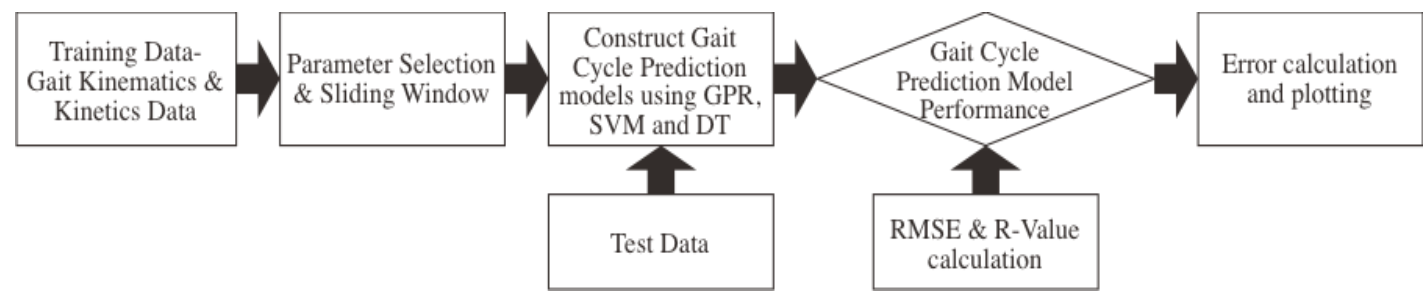

Figure 2. Prediction of gait cycle methodology

\subsection{Data preparation}

Dataset from the volunteers consisting of 24 young adults (age 27.6 \pm 4.4 years, height $171.1 \pm 10.5$ $\mathrm{cm}$, and mass $68.4 \pm 12.2 \mathrm{~kg}$ ) were retrieved from Fukuchi et al. [19]. The dataset contains kinematics data of joint angles and moment of the hip, knee, and ankle joints and of the pelvis and foot segments during overground walking at various speeds. Figure 3 shows joint angle of hip and knee and GRF at the sagittal, frontal, and transverse planes.

All gait trials were performed at three different speeds: comfortable speed, and then at a speed of $30 \%$ faster and a speed of 30\% slower than the comfortable speed. The dataset used as the parameters for this study are the volunteer information namely weight and height, joint angles of hip and knee, and GRF at sagittal plane. There are several techniques that have previously been used for gait cycle detection, for instance, using angle measurement using goniometers at hip, knee, and ankle joints [20] and ground reaction force measurement using pressure sensors [21].

The input parameters extracted from the dataset used in this study are weight $(\mathrm{Kg})$, height $(\mathrm{cm})$, hip angle (deg) and knee angle (degree) and one output data, which is the gait cycle (\%). Initially, the gait cycle prediction model is developed by using the current step of all the input parameters used. Then the model training data in each step was combined with one of the previous ones in order to better observe the effect of gait cycle in the model and enhance the accuracy. This approach is called sliding window technique, where the idea is to feed not only input parameters at current gait cycle, but also previous input parameters, thus it can incorporate auto correlation information into the model as suggested by Khairuddin et al. [22]. This way the variation in each input could be better counted for by the models. All input and output parameters are listed in Table 1. These input and output parameters are used as the equation variables for all machine learning techniques used in this study.
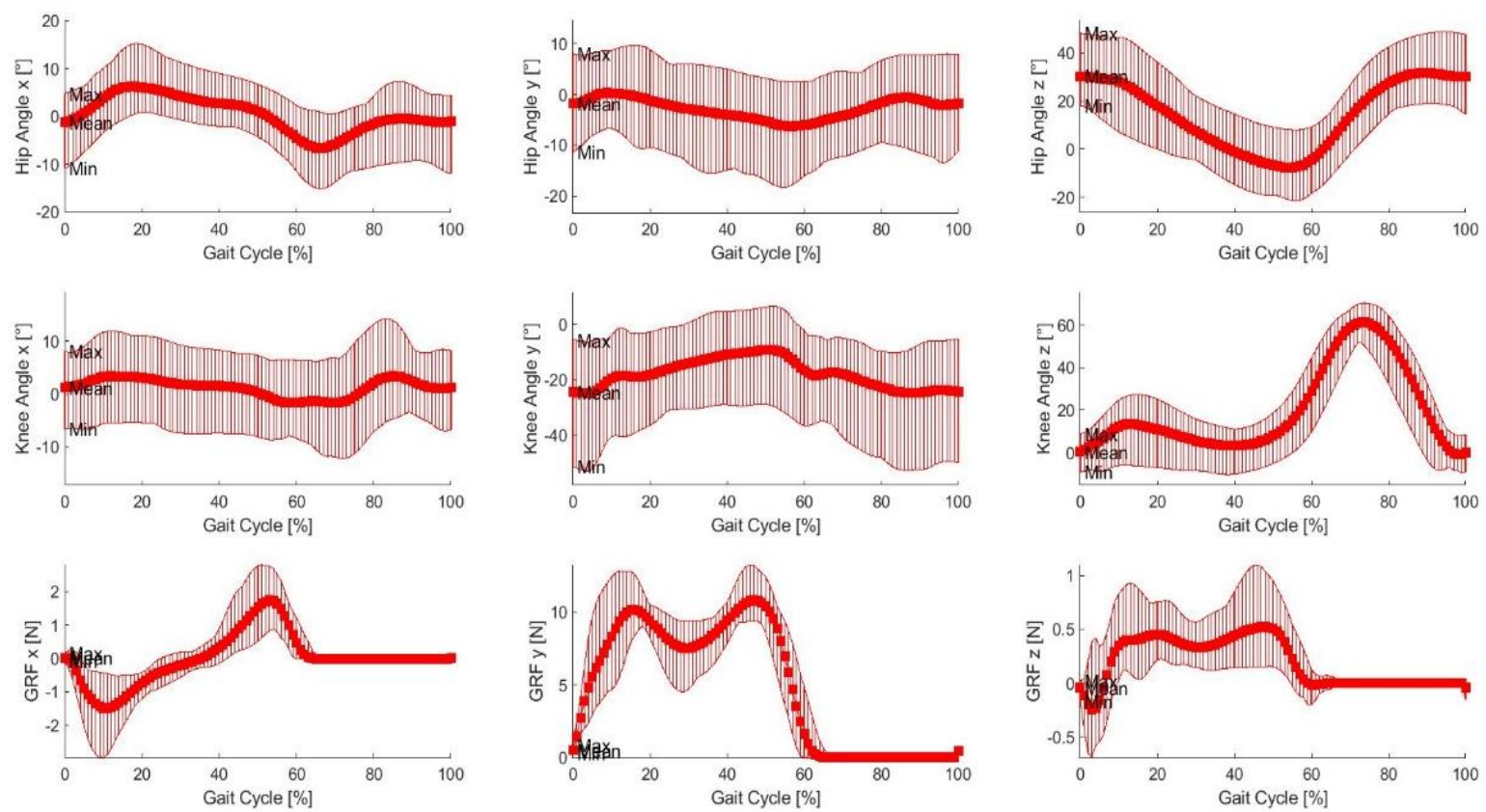

Figure 3. Joint angle of hip and knee and GRF at the frontal (x-axis), transverse (y-axis) and sagittal planes (z-axis) [19] 
Table 1. Input and output parameters to machine learning models

\begin{tabular}{cc} 
Input & Output \\
\hline Weight, Height, Hip Angle, Knee Angle, Ground Reaction Force & Gait Cycle \\
\hline
\end{tabular}

\subsection{Machine learning technique}

Machine learning (ML) discipline is a subset of artificial intelligence (AI) concerned with the ability of computer systems or machines to improve performance automatically throughout its input parameter during data training process [23]. ML algorithms were used to implement into the different models. According to recent study in prediction of using ML, it shows that Gaussian process regression (GPR), support vector machine (SVM) and decision tree (DT) yield advantage in speedup the ability to predict in generalization performance and in case of small sample [24]-[26]. Thus, by knowing the advantages of those ML algorithms we used GPR, SVM and DT in this study to develop the gait cycle prediction model.

\subsubsection{Gaussian process regression}

GPR method is a supervised machine learning method. GPR is completely specified by its mean function and covariance function. As explained by Quinonero-Candela et al. [27], the learning regressor is quantified in terms of the Bayesian estimation problem, for which predictive role is inferred from the deployment of an arbitrary procedure derived from the Gaussian distribution. Given a training input parameter data $x_{*}$ the best estimate of the output value, gait cycle, $f_{*}$ connected with it is represented by the anticipation of the anticipated output quantity to $\mathrm{L}$ and $x_{*}$ :

$$
\widehat{f}_{*} \mid L, x_{*} \sim E\left\{f_{*} \mid L, x_{*}\right\}=\int f_{*} p\left(f_{*} \mid L, x_{*}\right) d f_{*}
$$

The gait cycle predictive distribution $p\left(f_{*} \mid \mathrm{L}, x_{*}\right)$ can be shown in:

$$
p\left(f_{*} \mid L, x_{*}\right) \sim N\left(\mu_{*}, \sigma_{*}^{2}\right)
$$

where

$$
\begin{aligned}
& \mu_{*}=k_{*}^{t}\left[K+\sigma_{n}^{2} I\right]^{-1} \cdot y+b \\
& \sigma_{*}^{2}=\mathrm{k}\left(x_{*}, x_{*}\right)-k_{*}^{t}\left[K+\sigma_{n}^{2} I\right]^{-1} k_{*}
\end{aligned}
$$

And, given a covariance function $k\left(x, x^{\prime}\right), \mathrm{K}$ and $k_{*}$ represent training samples covariance matrix and covariance vector between training samples and sample $\mathrm{x} *$ respectively. In addition, $b, \sigma_{n}$ and $I$ are bias factor, noise variance, and identified matrix, respectively. It is indeed possible to restore two critical components from them: i) the mean $\mu_{*}$ which implies the best estimate of the output value according to (1) for the sample considered; ii) the variance $\sigma_{*}^{2}$ which reflects a confidence measurement linked to the output by the model. The covariance function $k\left(x, x^{\prime}\right)$ plays a pivotal role as it embeds the geometrical formation of the training samples. Squared exponential function is a normal choice for the covariance function:

$$
k\left(x, x^{\prime}\right)=\sigma_{f}^{2} \exp \left(-\frac{\left|x-x^{\prime}\right|}{2 l^{2}}\right)
$$

where, respectively, the two hyperparameters $\sigma_{f}^{2}$ and $l$ are described as process variance and length scale.

\subsubsection{Support vector machine (SVM)}

The training data $\left(x_{k}, y_{k}\right)_{k=1}^{I}$ in that, $x_{k} \in R^{n}$ is $\mathrm{n}$ dimension sample input, $y_{k} \in \mathrm{R}$ is the sample output. From nonlinear map function $\varphi(\cdot)$, the trained dataset was plotted non-linearly to a high dimension feature space (Hilbert space), then the nonlinear system recognition complexity was translated into a linear function that estimates the problem in a high dimension feature space. Complete formulation and deviation of SVM for regression can be found at [28]. Supposed the gait cycle prediction function as shown in (6).

$$
f(x)=\omega^{\mathrm{T}} \varphi\left(x_{k}\right)+b \omega \in R^{n h}, b \in R
$$

In the formula, the input space is plotted by nonlinear functions to an unclear dimension of feature space. The function estimation problem involves finding the minimum of the function $f(x)$, based on the structure risk minimization principle of Vapnik: 


$$
R_{\text {reg }}=\frac{1}{2}\|\omega\|^{2}+\mathrm{C} \cdot R_{e m p}^{t}
$$

where $\|\omega\|^{2}$ describes the complexity of model function $f(x)$, the constant $\mathrm{C}>0$ can be regulated to compromise between the error of the train and complexity of the model. The experiential risk:

$$
R_{e m p}^{t}=\frac{1}{I} \sum_{i=1}^{t}|y-f(x)|_{t}
$$

Insensitiveness loss function $|y=f(x)|_{\epsilon}$ is defined as (9):

$$
y-\left.f(x)\right|_{\epsilon}=\max \{0,|y-f(x)|-\varepsilon\}
$$

Then (7) minimum risk function is equal to optimizing the following problem. The object function:

$$
\min _{\omega b \xi \xi^{*}} J=\frac{1}{2}\|\omega\|^{2}+C \sum_{i=1}^{l}\left(\xi_{j}+\xi_{j}^{*}\right)
$$

The constraints: $\left\{\begin{array}{c}\sum_{i=1}^{I}\left(\alpha_{i}-\alpha_{i}^{*}\right)=0 \\ \alpha_{i}, \alpha_{i}^{*} \in[0, C]\end{array}\right.$. The output of support vector machine is:

$$
\left\{\begin{array}{c}
\omega=\sum_{i=1}^{n 5 v}\left(a_{i}-a_{i}^{*}\right)^{4}\left(x_{i}\right) \\
f(x)=\sum_{i=1}^{n s v}\left(a_{i}-a_{i}^{*}\right) k\left(x_{i}, x\right)+b
\end{array}\right.
$$

where $x_{i}$ in $\left(a_{i}-a_{i}^{*}\right) \neq 0$ is support vector, $a_{i}-a_{i}^{*}$ is support value, and $n s v$ is the number of support vector. The kernel function $k\left(x_{i}, x_{j}\right)=\varphi\left(x_{i}\right)^{T} \varphi\left(x_{j}\right)$ is an arbitrary symmetry function that is acceptable with the Mercer condition.

\subsubsection{Decision trees (DT)}

A DT is a recursive binary division into a set of rectangles for a future space. The tree is a simple function of the input in every rectangle; it is usually a constant. Therefore, the input-output map of a DT has the form of as refer from classification and regression tree [29] developed by Breiman in 1984 which the algorithm for DT:

$$
\mathcal{Y}=t(x)=\sum_{i=1}^{k} C_{i} I\left(x \in R_{i}\right.
$$

where $x$ is the input parameter data $\mathrm{N}$-dimensional vector, $x=\left(x_{1}, x_{2}, \ldots, x_{n}\right)$ and $I$ is the input indicator function which assumes a value of 1 if the input is true and 0 otherwise. Whenever the input $x$ is in rectangle $R_{i}$, the tree takes outputs the value $C_{i}$. Complex functions can be recognized by using a good enough partition. It is possible to extract the output of a DT very soon by performing a couple of observations. There is a pair $(j, \mathcal{S})$ at each node of the tree, where $j$ is the index of a variable $x_{j}$ in the input vector $x$, and $\mathcal{S}$ is the threshold. If $x_{j}<\mathcal{S}$, the tree is descends to the left, otherwise it descends to the right. In the tree, leaf nodes differ from regular nodes in that they store only a constant value. When a tree descends, the output is the constant value ci for that node upon reaching a leaf node. Although comparisons are made with the $x$ elements, the sides of the $R_{i}$ regions are parallel to the axes of the coordinates.

\subsection{Sliding window data (SWD)}

To improve the accuracy on the prediction of gait cycle, an SWD prediction scheme was proposed. A SWD is normally used to segment a data sequence [30], which in our study SWD on the input parameter of hip angle, knee angle and ground reaction force is used. This study used three different window size of 3 gait cycles for training and validation of the gait cycle prediction models. The SWD is represented in (13).

$$
\operatorname{SWD}=\left[\left(x_{t}, \widehat{y_{t}}\right),\left(x_{t}-1, \widehat{y_{t}}-1\right), \ldots,\left(x_{t}-\mathrm{N}-1, \widehat{y_{t}}-\mathrm{N}-1\right)\right]
$$

where $N$ is the sliding window width, $x$ and $y$ are output and output vectors, respectively. The input matrix is $x=\left[x_{t}, \ldots, x_{t}=N+1\right] \in R^{n_{x} \times N}$ and the output matrix is $y=\left[\hat{y}_{t}, \ldots, y_{t}-\widehat{N}+1\right]^{T} \in R^{n} y^{x} N$, respectively, where $n_{x}$ and $n_{y}$ are input and output dimensions. Data $x(t-N), x(t-N+1), \cdots, x(t-1)$ is the training 
input data at gait cycle $t$, and $x(t)$ is the gait cycle output data. The data at gait cycle $t+1$ is the value to be predicted after the training period, based on the current $x(t-N+1), x(t-N+2), \ldots, x(t)$ input update. Figure 4 can be used to express the scheme of SWD.

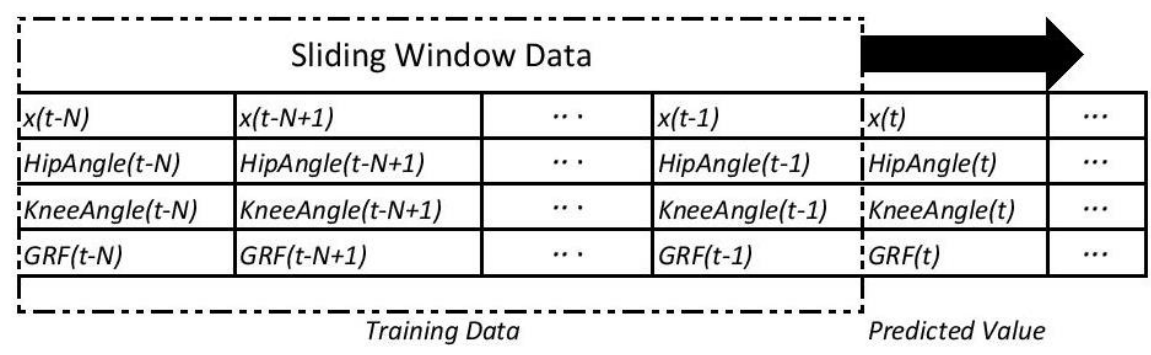

Figure 4. SWD

\subsection{Training and testing}

As according to study by Kumari et al. [31] and Shams-Baboli and Ezoji [32], the training and validation or testing dataset was set at $70 \%$ and $30 \%$ respectively, with good accuracy result. Hence, the dataset was divided into three part $70 \%$ of the total data was used for training, $15 \%$ used for validation, and the remaining $15 \%$ used for testing. In the case of small sample size data, a cross-validation method was used to evaluate the performance of the gait cycle prediction model. A five-fold cross-validation scheme, that is, 24 young adult results, was divided into five segments. Firstly, the prediction model was trained and built using four out of the five segments, and the remaining one was used for validation. Secondly, the procedures described above have been replicated five times. Finally, to achieve a final output result, the five prediction results were averaged. The training and validation were carried out with four different SWD conditions that are i) $x(\mathrm{t})$, ii) $x(\mathrm{t}-1)$, iii) $x(\mathrm{t}-2)$ and iv) $x(\mathrm{t}-3)$. The RMSE and $\mathrm{R}-$ Value were used to measure the correlation between predicted gait cycle by each machine learning technique and the actual target values. These two criteria, RMSE and R-Value, were used as the basis for training and selecting the idealized prediction model. RMSE was calculated based on actual value and those predicted by GPR, SVM and DT model, respectively, using:

$$
\mathrm{RMSE}=\sqrt{\left(\sum\left(\hat{y} i-y_{i}\right)^{2}\right) N}
$$

and R-Value can be presented as (15).

$$
R^{2}=1-\frac{\Sigma_{i}\left(y_{i}-\hat{y}_{i}\right)^{2}}{\Sigma_{i}\left(y_{i}-\bar{y}\right)^{2}}
$$

where $\hat{y}_{\mathrm{i}}=$ predicted value, $y_{i}=$ actual value, $\bar{y}=$ is mean of the actual value and $\mathrm{N}=$ number of measured data points.

\section{RESULTS AND DISCUSSION}

In this section, the training, validation, and testing for the gait cycle prediction model are discussed. As discussed in the methodology section, different types of machine learning technique GPR, SVM and DT are used to develop gait cycle prediction model. The model is with and without the enhanced SWD, and the RMSE and R-Value results are compared and discussed. Then, the gait cycle prediction model is tested with the RMSE and R-Value results are compared from subjects' data that was not used during the training and validation process.

\subsection{Training and validation: Gait cycle prediction model using GPR, SVM and DT}

We applied GPR, SVM and DT to gait cycle prediction using joint angles of hip and knee and GRF dataset of humans during normal gait without any additional input parameter of SWD technique. The training and validation results with different types of machine learning technique GPR, SVM and DT are summarized in Table 2. Results are expressed in terms of RMSE and R-Value for estimation of which is the best model to be used. The results on GPR, SWM and DT training show that for RMSE and R-Value of 13.297 and 0.78, 14.059 and 0.77 , and 11.535 and 0.84 for GPR, SVM and DT, respectively. Over the three models validated 
here, SVM and GPR models had similar performance because they are exemplar-based kernel machines. In addition, study by Hultquist et al. [33] and Nguyen-Tuong and Peters [34] shows that SVM often gives a sparse model that is fitted only from a subset of training samples, whereas GPR is a full model based on all the training samples. At this stage, DT model had the highest performance result compared to GPR and SVM model, where the prediction result is shown in Figure 5. The performance result for GPR in Figure 5(a) and SVM in Figure 5(b) was low as compared to DT model in Figure 5(c), because at gait cycle $0 \%-10 \%$ and $90 \%-100 \%$, the prediction did not fitted nearly to the accurate prediction level. However, from $10 \%-90 \%$ of gait cycle, the prediction fitted at the accurate prediction level, indicating that all GPR, SVM and DT model have good performance for gait cycle prediction.

Table 2. Result on different training model for gait cycle prediction

\begin{tabular}{ccccccc}
\hline & \multicolumn{3}{c}{ Training } & \multicolumn{3}{c}{ Validation } \\
& GPR & SVM & DT & GPR & SVM & DT \\
\hline RMSE & 13.297 & 14.059 & 11.535 & 14.983 & 14.665 & 12.234 \\
R-Value & 0.78 & 0.77 & 0.84 & 0.76 & 0.76 & 0.82 \\
\hline
\end{tabular}

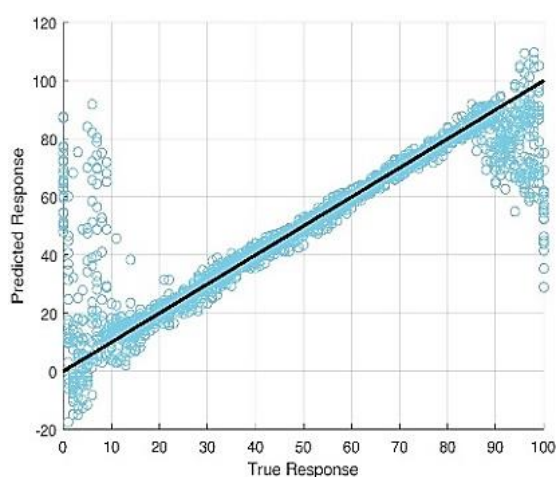

(a)

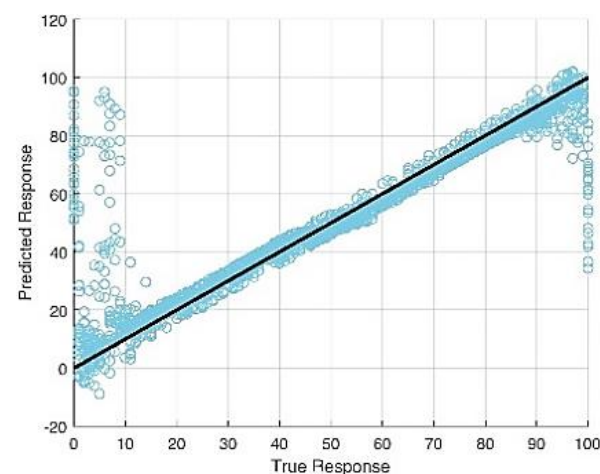

(b)

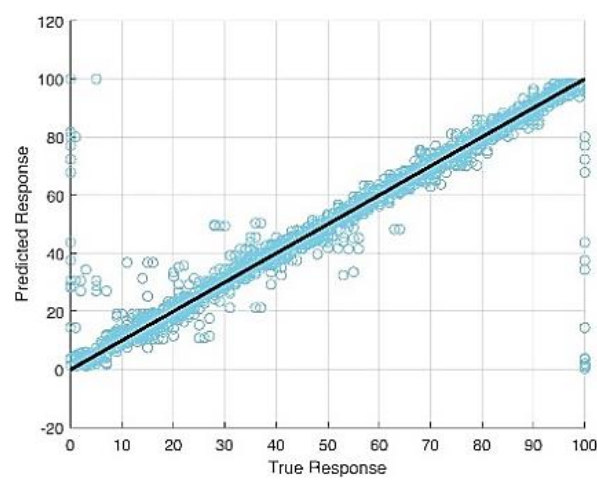

(c)

Figure 5. Model prediction result without SWD, straight line indicates accurate prediction level; (a) GPR model, (b) SVM model, (c) DT model

\subsection{Training and validation: Gait cycle prediction model enhancement using SWD}

The training results with different types of sliding window $(t=0, t-1, t-2, t-3)$ data are summarized in Table 3. The results for sliding window $x(t-3)$ for GPR, SWM and DT models are shown in Figure 6 and gait cycle prediction accuracy with RMSE and R-Value of each machine learning technique are presented in Table 4. For all the training models, the RMSE and R-Value decreased when the sliding window of input data increased. This is because more previous information can be fed into the training model so that it will enhance the accuracy in the prediction gait cycle. Among the three different models, DT model gives good results with accuracy of 11.535 (RMSE) and 0.84 (R-Value) at $x(t=0)$ and 3.7769 (RMSE) and 0.98 (RValue) at $x(t-3)$. From the training results also, during sliding window $x(t-3)$, all the models show good accuracy with 9.3034 and $0.90,7.3247$ and 0.94 , and 3.7769 and 0.98 for RMSE and R-Value for SVM, GPR and DT model, respectively. In GPR and SVM model, as according to Figure 6(a) and Figure 6(b), the predicted 
response during early $10 \%$ and late $90 \%$ had improved compared with the results without SWD, as shown in Figure 5(a) and Figure 5(b). However, from 10\% to $90 \%$ gait cycle the result showed a good prediction level as it fitted near to the accurate prediction level. In contrast, for DT model as shown in Figure 6(c), the predicted response fitted evenly along the accurate prediction level. Thus, the best model for gait cycle prediction to be used during testing was the DT model.

Table 3. Result on different training model for different SWD

\begin{tabular}{cccccc}
\hline & & \multicolumn{3}{c}{ SWD } & \\
& & $\mathrm{t}=0$ & $\mathrm{t}-1$ & $\mathrm{t}-2$ & $\mathrm{t}-3$ \\
\hline GPR & RMSE & 13.297 & 7.4239 & 7.4391 & 7.3247 \\
& R-Value & 0.78 & 0.93 & 0.93 & 0.94 \\
SVM & RMSE & 14.059 & 9.7712 & 10.32 & 9.3034 \\
& R-Value & 0.77 & 0.87 & 0.89 & 0.90 \\
DT & RMSE & 11.535 & 5.5761 & 5.4101 & 3.7769 \\
& R-Value & 0.84 & 0.96 & 0.97 & 0.98 \\
\hline
\end{tabular}

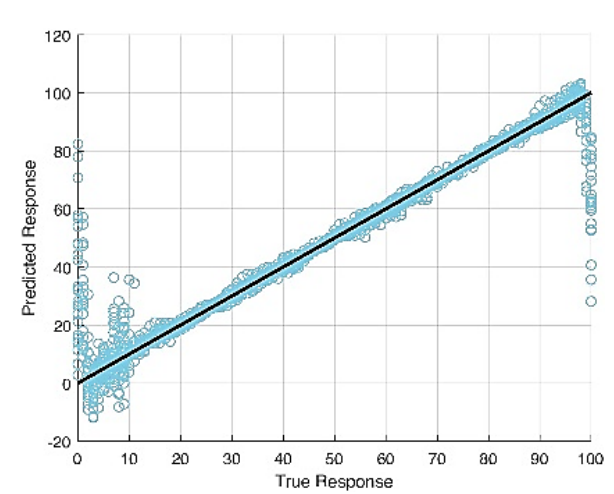

(a)

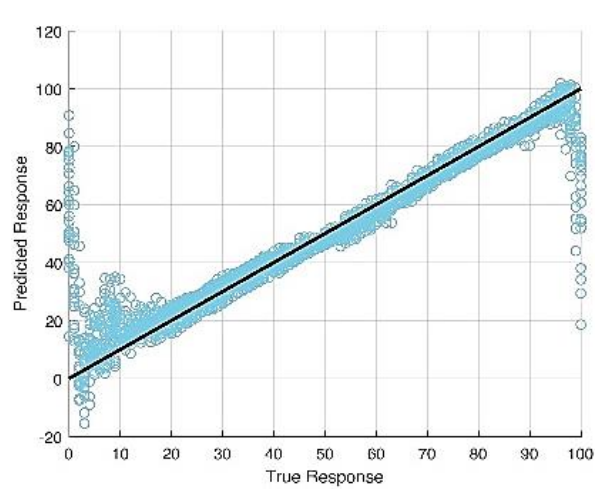

(b)

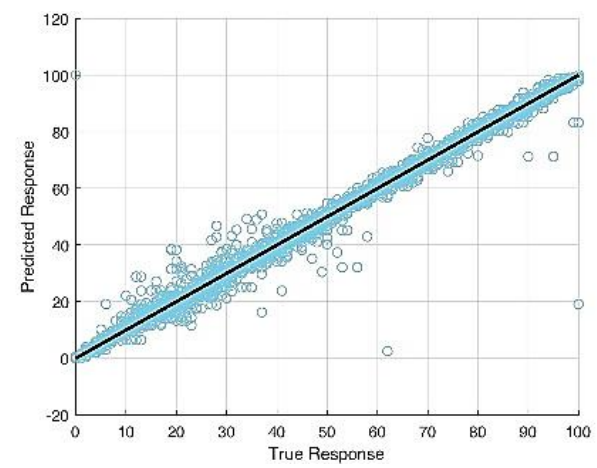

(c)

Figure 6. Model prediction result with SWD, straight line indicate accurate prediction level; (a) GPR model, (b) SWM model, (c) DT model

Table 4. Accuracies of gait cycle prediction

\begin{tabular}{ccccc}
\hline & \multicolumn{2}{c}{ Training } & \multicolumn{2}{c}{ Validation } \\
& RMSE & R-Value & RMSE & R-Value \\
\hline SVM & 9.3034 & 0.90 & 9.8183 & 0.89 \\
GPR & 7.3247 & 0.93 & 7.5665 & 0.92 \\
DT & 3.7769 & 0.98 & 3.3018 & 0.97 \\
\hline
\end{tabular}

Dey et al. [35] used minimum and maximum angles and moment of the hip, knee, and angle joints to predict values of gait pattern at different speed. The author has used quadratic regression technique in the study. Eslamy and Schilling [36] reported an R-Value score above 0.92 using GPR for ankle kinematic prediction for the trained speed levels. Dey et al. [37] reported R-Value of 0.98 for prediction of ankle joint angles by using SVM for level ground walking at self-selected normal speeds. However, the variation in the range of input features, the difference in data sets, the volume of data used and the different output quantification measures 
make it difficult to equate directly with other studies. However, none of these studies have attempted to model gait cycles using machine learning regression algorithms for assistive rehabilitation device control.

\subsection{Testing: Gait cycle prediction model}

Each of the models were tested using the testing data with four subjects $(n=6,10,13,19)$. The results are summarized in Table 5, and Figure 7(a) and Figure 7(b) show the GPR, SVM and DT complete gait cycle prediction testing results for subjects $n=6$ and 10, respectively. From the results, all the model developed reached good accuracies in which low RMSE value was at around 3 to 7 and high R-Value which was at 0.9 and above. Among the results, the DT model showed consistency of RMSE and R-Value which were around 3.0 and 0.95, respectively. Figure 7(a) and Figure 7(b) shows the GPR and SVM model prediction which did not perform well as the graph line divert from the actual line. In contrast the DT model showed very good results and fitted to the actual line, thus it showed that gait cycle prediction was best using the DT model.
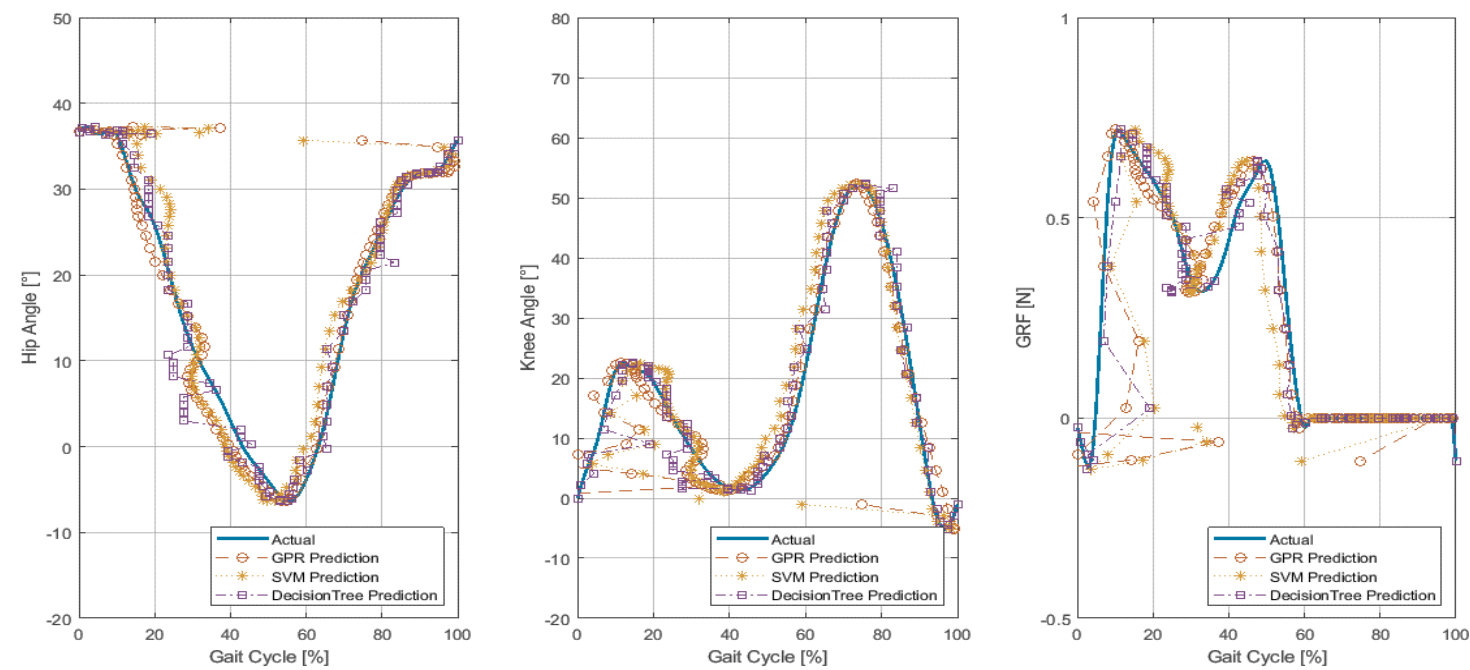

(a)
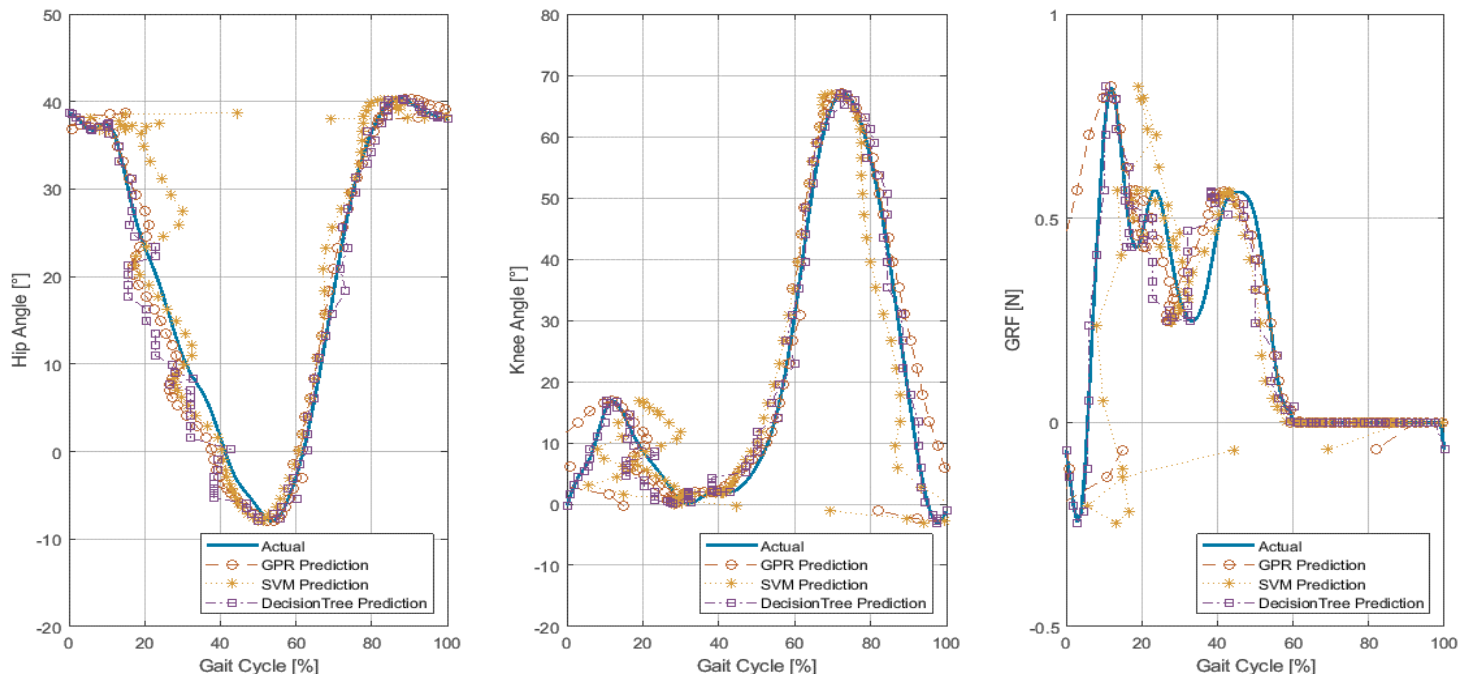

(b)

Figure 7. Model testing result, straight line indicates accurate prediction level; (a) for subject 6 and (b) for subject 10

This study demonstrates that ML with input parameter GRF, hip and knee angle can be used to predict human gait cycle. Unlike [24], [38] on joint moment prediction using ANN model, this study avoids the use or marker trajectories that could be time-consuming and complex equipment requires. It also effectively reduces 
the number of input parameters, which makes it possible to predict gait cycle. Our methods of SWD further enhance high accuracy of prediction with R-Value $>0.95$. The proposed method is therefore sufficient to be used as a model of the gait cycle for the design of control system for the assistive rehabilitation device.

Table 5. Testing result of accuracies for each model

\begin{tabular}{ccccccc}
\hline \multirow{2}{*}{ Subject No } & \multicolumn{2}{c}{ GPR } & \multicolumn{2}{c}{ SVM } & \multicolumn{2}{c}{ DT } \\
& RMSE & R-Value & RMSE & R-Value & RMSE & R-Value \\
\hline 6 & 5.8546 & 0.9597 & 7.4258 & 0.9351 & 3.6422 & 0.9844 \\
10 & 5.0207 & 0.9703 & 7.3810 & 0.9359 & 3.4768 & 0.9858 \\
13 & 7.1533 & 0.9597 & 11.9302 & 0.8326 & 3.6422 & 0.9678 \\
19 & 5.002 & 0.9706 & 7.8896 & 0.9268 & 3.7942 & 0.9831 \\
\hline
\end{tabular}

\section{CONCLUSION}

This paper presents gait cycle prediction models developed using machine learning techniques. The DT model is presents suitable for modeling of gait cycle using input parameters of height, weight, hip, and knee angle, and GRF. The gait cycle prediction model is enhanced further and achieved a better accuracy once the sliding window was introduced. Further investigations with input parameters such as joint moment to predict joint angle should be explored for further studies. This research would also benefit from assistive rehabilitation devices for gait cycle prediction.

\section{ACKNOWLEDGEMENTS}

This research was supported by Universiti Teknologi MARA, Cawangan Pulau Pinang for the funding of research grant from the Ministry of Education Malaysia (MOHE). The authors are grateful to the university and MOHE for supporting the present work.

\section{REFERENCES}

[1] M. Ismail, C. Lam, K. Sundaraj, and M. Rahiman, "Hand motion pattern recognition analysis of forearm muscle using mmg signals," Bulletin of Electrical Engineering and Informatics, vol. 8, no. 2, pp. 533-540, 2019, doi: 10.11591/eei.v8i2.1415.

[2] A. S. Yusof, A. I. Che-Ani, Z. Hussain, N. Hamzah, R. Boudville and M. F. A. Rahman, "Back-drivability of powered knee orthosis for knee free swing and knee extension," in 2017 th IEEE International Conference on Control System, Computing and Engineering (ICCSCE), Penang, Malaysia, 2017, pp. 331-335, doi: 10.1109/ICCSCE.2017.8284429.

[3] A. A. Abd. Rahim, A. I. Che-Ani, Z. Hussain, R. Boudville and K. A. Ahmad, "Development of Powered Knee Orthosis for FES-Assisted Knee Swing," in 2019 9th IEEE International Conference on Control System, Computing and Engineering (ICCSCE), Penang, Malaysia, 2019, pp. 209-214, doi: 10.1109/ICCSCE47578.2019.9068583.

[4] E. Noorsal, S. Yahaya, Z. Hussain, R. Bouldville, M. Ibrahim, and Y. Mohd Ali, "Analytical study of flexible stimulation waveforms in muscle fatigue reduction," International Journal of Electrical and Computer Engineering, vol. 10, no. 1, pp. 690-703, 2020, doi: 10.11591/ijece.v10i1.pp690-703.

[5] R. Boudville, Z. Hussain, S. Z. Yahaya, M. F. Abd Rahman, K. A. Ahmad, and N. I. Husin, "Development and Optimization of PID Control for FES Knee Exercise in Hemiplegic Rehabilitation," in 2018 12th International Conference on Sensing Technology (ICST), Limerick, Ireland, 2018, pp. 143-148, doi: 10.1109/ICSensT.2018.8603628.

[6] M. Akhtaruzzaman, A. A. Shafie, and M. R. Khan, "Gait analysis: Systems, technologies, and importance," Journal of Mechanics in Medicine and Biology, vol. 16, no. 7, p. 1630003, 2016, doi: 10.1142/S0219519416300039.

[7] F. Chen, X. Cui, Z. Zhao, D. Zhang, C. Ma, X. Zhang, and H. Liao, "Gait acquisition and analysis system for osteoarthritis based on hybrid prediction model," Computerized Medical Imaging and Graphics, vol. 85, Art. No. 101782, 2020, doi: 10.1016/j.compmedimag.2020.101782.

[8] M. Galli et al., "Use of the gait profile score for the quantification of the effects of robot-assisted gait training in patients with Parkinson's disease," 2016 IEEE 2nd International Forum on Research and Technologies for Society and Industry Leveraging a better tomorrow (RTSI), Bologna, Italy, 2016, pp. 1-4, doi: 10.1109/RTSI.2016.7740603.

[9] H. Vallery, E. H. F. van Asseldonk, M. Buss and H. van der Kooij, "Reference Trajectory Generation for Rehabilitation Robots: Complementary Limb Motion Estimation," in IEEE Transactions on Neural Systems and Rehabilitation Engineering, vol. 17, no. 1, pp. 23-30, Feb. 2009, doi: 10.1109/TNSRE.2008.2008278.

[10] T. X. Tran, C. -k. Kang and S. L. Mathis, "Lower-Gait Tracking Mobile Application: A Case Study of Lower body Motion Capture Comparison Between Vicon T40 System and Apple Augmented Reality," 2020 IEEE International Conference on Bioinformatics and Biomedicine (BIBM), 2020, pp. 2654-2656, doi: 10.1109/BIBM49941.2020.9313201.

[11] M. Ye, Q. Zhang, L. Wang, J. Zhu, R. Yang, and J. Gall, "A survey on human motion analysis from depth data," in Time-of-flight and depth imaging, sensors, algorithms, and applications. Springer, 2013, pp. 149-187, doi: 10.1007/978-3-642-44964-2_8. 
[12] E. Auvinet, J. Meunier and F. Multon, "Multiple depth cameras calibration and body volume reconstruction for gait analysis," in 2012 11th International Conference on Information Science, Signal Processing and their Applications (ISSPA), Montreal, QC, Canada, 2012, pp. 478-483, doi: 10.1109/ISSPA.2012.6310598.

[13] M. O. Derawia, "Derawi biometrics: Research on different biometrics modalities," http://biometrics. derawi.com/?page id=38, Feb. 2020.

[14] J. D. Farah, N. Baddour and E. D. Lemaire, "Gait phase detection from thigh kinematics using machine learning techniques," 2017 IEEE International Symposium on Medical Measurements and Applications (MeMeA), Rochester, MN, USA, 2017, pp. 263-268, doi: 10.1109/MeMeA.2017.7985886.

[15] M. Mahdavian, S. Arzanpour and E. J. Park, "Motion Generation of a Wearable Hip Exoskeleton Robot Using Machine Learning-Based Estimation of Ground Reaction Forces and Moments," in 2019 IEEE/ASME International Conference on Advanced Intelligent Mechatronics (AIM), Hong Kong, China, 2019, pp. 796-801, doi: 10.1109/AIM.2019.8868759.

[16] Susanto, R. Analia and K. Song, "Design of assistive torque for a lower limb exoskeleton based on motion prediction," in 2016 55th Annual Conference of the Society of Instrument and Control Engineers of Japan (SICE), Tsukuba, Japan, 2016, pp. 172-177, doi: 10.1109/SICE.2016.7749261.

[17] A. J. Young, A. Simon, and L. J. Hargrove, "An intent recognition strategy for transfemoral amputee ambulation across different locomotion modes," in 2013 35th Annual International Conference of the IEEE Engineering in Medicine and Biology Society (EMBC), Osaka, Japan, 2013, pp. 1587-1590, doi: 10.1109/EMBC.2013.6609818.

[18] L. Kilmartin, R. K. Ibrahim, E. Ambikairajah, and B. Celler, "Optimising recognition rates for subject independent gait pattern classification,” IET Irish Signals and Systems Conference (ISSC 2009), Dublin, Ireland, 2009, pp. 1-6.

[19] C. A. Fukuchi, R. K. Fukuchi, and M. Duarte, "A public dataset of overground and treadmill walking kinematics and kinetics in healthy individuals," PeerJ, vol. 6, no. 9, Art. No. e4640, 2018, doi: 10.7717/peerj.4640.

[20] San Kuen Ng and H. J. Chizeck, "Fuzzy model identification for classification of gait events in paraplegics," in IEEE Transactions on Fuzzy Systems, vol. 5, no. 4, pp. 536-544, Nov. 1997, doi: 10.1109/91.649904.

[21] H. Wu, Z. Zhou, J. Wang, H. An, and Q. Wei, "Recognization of Stance Phase Using Flexible Pressure Sensors," in 2016 8th International Conference on Intelligent Human-Machine Systems and Cybernetics (IHMSC), Hangzhou, China, 2016, pp. 509-512, doi: 10.1109/IHMSC.2016.218.

[22] I. A. Khan, A. Akber and Y. Xu, "Sliding Window Regression based Short-Term Load Forecasting of a Multi-Area Power System," in 2019 IEEE Canadian Conference of Electrical and Computer Engineering (CCECE), Edmonton, AB, Canada, 2019, pp. 1-5, doi: 10.1109/CCECE.2019.8861915.

[23] A. Khairuddin, K. Ku Azir, and P. Eh Kan, "A general framework for improving electrocardiography monitoring system with machine learning," Bulletin of Electrical Engineering and Informatics, vol. 8, no. 1, pp. 261-268, 2019, doi: 10.11591/eei.v8i1.1400.

[24] Z. Yin and Jian Hou, "Recent advances on svm based fault diagnosis and process monitoring in complicated industrial processes," Neurocomputing, vol. 174, pp. 643-650, 2016, doi: 10.1016/j.neucom.2015.09.081.

[25] H. Lu, N. Zou, R. Jacobs, B. Afflerbach, X. Lu, and D. Morgan, "Error assessment and optimal cross-validation approaches in machine learning applied to impurity diffusion," Computational Materials Science, vol. 169, Art. No. 109075, 2019, doi: 10.1016/j.commatsci.2019.06.010.

[26] J. Ling, M. Hutchinson, E. Antono, S. Paradiso, and B. Meredig, "High-Dimensional Materials and Process Optimization Using Data-Driven Experimental Design with Well-Calibrated Uncertainty Estimates," Integrating Materials and Manufacturing Innovation, vol. 6, no. 3, pp. 207-217, 2017, doi: 10.1007/s40192-017-0098-z.

[27] J. Quinonero-Candela, C. E. Rasmussen, and C. K. Williams, "Approximation methods for Gaussian process regression," in Large-scale kernel machines. MIT Press, 2007, pp. 203-223.

[28] A. J. Smola and B. Scholkopf, "A tutorial on support vector regression," Statistics and computing, vol. 14, no. 3, pp. 199-222, 2004, doi: 10.1023/B:STCO.0000035301.49549.88.

[29] L. Breiman, J. Friedman, C. J. Stone, and R. A. Olshen, "Classification and regression trees," CRC press, 1984.

[30] I. P. E. S. Putra and R. Vesilo, "Window-size impact on detection rate of wearable-sensor-based fall detection using supervised machine learning," in 2017 IEEE Life Sciences Conference (LSC), Sydney, NSW, Australia, 2017, pp. 21-26, doi: 10.1109/LSC.2017.8268134.

[31] C. U. Kumari et al., "Heart Rhythm Abnormality Detection and Classification using Machine Learning Technique," in 2020 4th International Conference on Trends in Electronics and Informatics (ICOEI)(48184), Tirunelveli, India, 2020, pp. 580-584, doi: 10.1109/ICOEI48184.2020.9142914.

[32] A. Shams-Baboli and M. Ezoji, "A Zernike moment based method for classification of Alzheimer's disease from structural MRI," in 2017 3rd International Conference on Pattern Recognition and Image Analysis (IPRIA), Shahrekord, Iran, 2017, pp. 38-43, doi: 10.1109/PRIA.2017.7983061.

[33] C. Hultquist, G. Chen, and K. Zhao, "A comparison of Gaussian process regression, random forests and support vector regression for burn severity assessment in diseased forests," Remote sensing letters, vol. 5, no. 8, pp. 723-732, 2014, doi: 10.1080/2150704X.2014.963733.

[34] D. Nguyen-Tuong and J. Peters, "Local Gaussian process regression for real-time model-based robot control," in 2008 IEEE/RSJ International Conference on Intelligent Robots and Systems, Nice, France, 2008, pp. 380-385, doi: 10.1109/IROS.2008.4650850.

[35] S. Dey, T. Yoshida, and A. F. Schilling, "Feasibility of training a random forest model with incomplete user-specific data for devising a control strategy for active biomimetic ankle," Frontiers in Bioengineering and Biotechnology, vol. 8, pp. 855 , 2020, doi: 10.3389/fbioe.2020.00855. 
[36] M. Eslamy and A. F. Schilling, "A Conceptual High Level Controller to Walk with Active Foot Prostheses/Orthoses," in 2018 7th IEEE International Conference on Biomedical Robotics and Biomechatronics (Biorob), Enschede, 2018, pp. 1224-1229, doi: 10.1109/BIOROB.2018.8487213.

[37] S. Dey, M. Eslamy, T. Yoshida, M. Ernst, T. Schmalz and A. Schilling, "A Support Vector Regression Approach for Continuous Prediction of Ankle Angle and Moment During Walking: An Implication for Developing a Control Strategy for Active Ankle Prostheses," in 2019 IEEE 16th International Conference on Rehabilitation Robotics (ICORR), Toronto, ON, Canada, 2019, pp. 727-733, doi: 10.1109/ICORR.2019.8779445.

[38] W. De Vries, D. Veeger, C. Baten, and F. van der Helm, "Can shoulder joint reaction forces be estimated by neural networks?," Journal of Biomechanics, vol. 49, no. 1, pp. 73-79, 2015, doi: 10.1016/j.jbiomech.2015.11.019.

\section{BIOGRAPHIES OF AUTHORS}
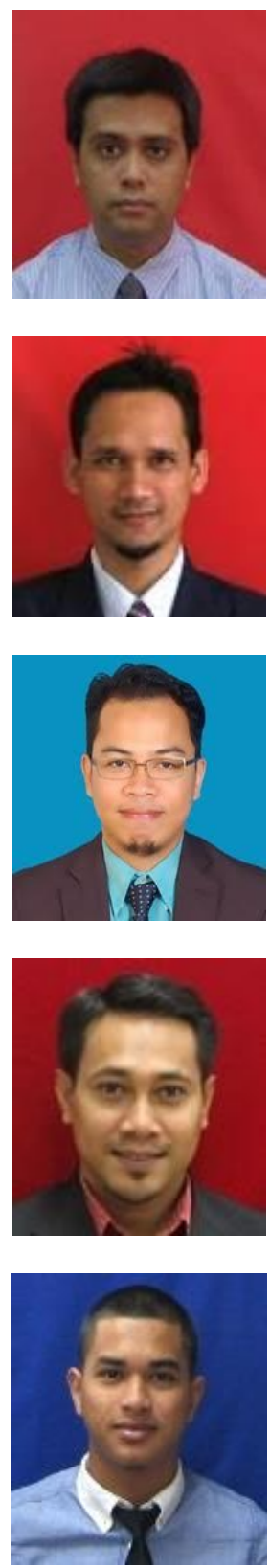

Adi Izhar Che Ani is a Senior Lecturer at Faculty of Electrical Engineering, Universiti Teknologi MARA Cawangan Pulau Pinang (UiTMCPP) with a Master's Degree in Engineering from Universiti Malaya Malaysia (2012). He obtained his Bachelor's Degree in Electrical \& Electronics Engineering from University of Miyazaki (Japan) in 2007. His research interests are the fields of biomedical engineering that focus on modeling and control of assistive device for human gait rehabilitation. Recently, he is at the doctoral candidate specializing in the area human gait rehabilitation.

Assoc. Prof. Ir. Dr. Zakaria Hussain obtained his Bachelor's Degree in Electrical and Electronics Engineering from The University of Huddersfield, United Kingdom in 1997 and he obtained a Doctoral degree in Automatic Control and System Engineering from The University of Sheffield, United Kingdom in 2010. His main research interests include Intelligent Control Technique, Rehabilitation Engineering and Artificial Intelligence. He is currently a Lecturer at the Faculty of Electrical Engineering, Universiti Teknologi Mara Cawangan Pulau Pinang (UiTMCPP), Malaysia.

Mohd Ikmal Fitri Maruzuki received his B.Eng. in Computer Engineering from Ehime University (Japan) in 2004 and his Master's degree in Communication and Computer Engineering from Universiti Kebangsaan Malaysia (UKM) in 2010. He is currently employed as a Lecturer at the Faculty of Electrical Engineering in Universiti Teknologi MARA Cawangan Pulau Pinang (UiTMCPP) Malaysia. His research interests include on machine learning, image processing and embedded systems.

Ts. Dr. Mohd Suhaimi Sulaiman had received his Ph.D. in Electrical Engineering from Universiti Teknologi MARA Cawangan Pulau Pinang (UiTMCPP), Malaysia in 2019. Currently he is a senior lecturer at the Faculty of Electrical Engineering at UiTMCPP. His research interests include classification in artificial neural network, agrotechnology and electrical measurement system. His Ph.D. research title is 'White Root Disease Auto-Detection System for Rubber Trees Based on Dynamic Electro-Biochemical Latex Properties'.

Ahmat Adam Abd Rahim is currently pursuing a master's degree in electrical engineering at Universiti Teknologi MARA (UiTM) Shah Alam. He obtained his Bachelor's Degree in Electrical and Electronic Engineering in 2019. His area of interest includes Intelligent Control Technique, Rehabilitation Engineering and Finite Element Analysis. 